\title{
LES DOMAINES MAGNÉTIQUES
}

\author{
P. BRISSONNEAU \\ Institut Polytechnique de Grenoble \\ E. N. S. d'Electrotechnique et Génie Physique, B. P. no 15, 38040 Grenoble Cedex, France
}

\begin{abstract}
Résumé. - On considère un modèle de moments localisés et on passe en revue les différentes énergies qui interviennent pour déterminer l'orientation des moments: l'énergie d'échange, l'énergie magnétostatique, l'anisotropie magnétocristalline, les effets magnétoélastiques, et enfin l'énergie de couplage avec les défauts de réseau.

Tout échantillon ferromagnétique se divise spontanément en domaines d'aimantation uniforme pour abaisser l'énergie totale du système des moments, deux domaines adjacents étant séparés par une couche de transition appelée une paroi.

Après un exposé général des principales méthodes utilisées pour l'observation des domaines, on explique comment la structure en domaines intervient dans les processus d'aimantation.

Abstract. - A review is presented of the various kinds of energies occurring to determine the arrangement of the localised magnetic moments : the exchange energy, the magnetostatic energy, the magnetocrystalline anisotropy, the magnetoelastic effects, and the coupling energy with lattice defects.

Then it results that any ferromagnetic sample divides itself spontaneously in domains of uniform magnetization with the object of decreasing the total energy of the moments system, two adjacent domains being separated by a transition region called a wall.

We examine the main technics used to observe the domains and the effects of the general configuration on the magnetization processes.
\end{abstract}

Introduction. - Pour analyser le comportement d'un bon nombre de matériaux magnétiques, il est souvent utile et parfois indispensable de tenir compte de l'existence des domaines magnétiques. L'exposé qui suit, destiné à un public de non-spécialistes, se limite aux aspects considérés comme classiques. On essaie d'expliquer, le plus simplement possible, pourquoi il y a des domaines magnétiques et comment cette structure, à une échelle intermédiaire entre l'échelle atomique et l'échelle macroscopique, rend compte des aspects parmi les plus originaux du comportement des matériaux magnétiques.

1. Le modèle microscopique. - A l'échelle atomique on peut concevoir un solide ferro- ou ferrimagnétique comme un assemblage régulier de moments magnétiques localisés, c'est-à-dire attachés à chacun des atomes ou des ions qui composent le cristal, et caractéristiques de sa structure électronique. Toutes les influences extérieures, un champ magnétique appliqué par exemple, ou encore l'agitation thermique, n'entraînant que des modifications négligeables de la structure électronique ne peuvent que provoquer des rotations des moments.

Toutes les propriétés d'un cristal, l'aimantation à saturation, l'hystérésis, l'aimantation rémanente ne sont que le reflet des rotations des $N$ moments atomiques qui le composent. Avec un tel modèle on se trouve alors devant un problème à $2 N$ degrés de liberté.

2. Les différentes espèces d'énergie. - Pour comprendre comment s'orientent les moments atomiques, il faut commencer par recenser toutes les énergies mises en jeu dans un tel système. Nous allons suivre un exposé proposé initialement par C. Kittel [1]. Par ordre d'importance décroissante, on trouve :

2.1 L'INTERACTION D'ÉCHANGE RESPONSABLE DU FERRO- OU DU FERRIMAGNÉTISME. - DeuX moments magnétiques voisins tendent à s'aligner soit parallèlement dans un ferromagnétique, soit antiparallèlement dans un antiferromagnétique. S'il n'y avait en jeu que ce type d'interaction on observerait un alignement parfait des $N$ moments qui composent le cristal et le moment résultant serait en permanence le moment à saturation.

On peut admettre dans un grand nombre de cas que cette interaction provient d'un Hamiltonien de spin, qu'on appelle l'Hamiltonien d'Heisenberg :

$$
\mathscr{H}=-\sum_{\mathbf{i} \neq \mathbf{j}} J_{\mathbf{i j}} \mathbf{S}_{\mathbf{i}} \cdot \mathbf{S}_{\mathbf{j}}
$$

où $\mathbf{S}_{\mathbf{i}}$ est le moment de spin de l'atome $\mathrm{i}, \mathbf{S}_{\mathbf{j}}$ celui de l'atome $\mathrm{j}$ et $J_{\mathrm{ij}}$ l'intégrale d'échange entre les atomes $\mathrm{i}$ et $\mathrm{j}$. $J_{\mathrm{ij}}$ dépend de la distance entre les porteurs i et $\mathrm{j}$ et décroît rapidement si cette distance augmente. On connaît aussi des cas où $J_{\mathrm{ij}}$ est une fonction oscillante. Quand $J_{\mathbf{i j}}$ est positif, par effet coopératif tous les moments de spin tendent à s'aligner et on a un cristal ferromagnétique. Au contraire $J_{\mathbf{i j}}<0$ correspond au cas ferri- et antiferromagnétique.

Quand le parallélisme des moments n'est plus assuré, il est important de noter que l'équation (2.1) traduit une interaction variant comme $\cos \varphi_{\mathbf{i j}}$ entre les spins $\mathrm{i}$ et $\mathrm{j}$, c'est-à-dire très lentement autour des valeurs $\varphi_{i j}=0$ et $\pi$. En d'autres termes, de petits écarts au parallélisme n'augmentent qu'assez peu l'énergie du système bien que $J_{\mathbf{i j}}$ soit toujours important. L'énergie d'échange a donc surtout pour effet 
d'interdire à l'échelle atomique toute variation brutale d'orientation des moments.

2. 2 L'ÉNERGIE MAGNÉTOSTATIQUe. - On désigne ainsi l'énergie de couplage qui résulte des interactions coulombiennes entre les moments magnétiques. Chaque moment élémentaire étant placé dans un champ local dû à tous ses voisins, il en résulte une énergie magnétostatique :

$$
U_{\mathrm{m}}=-\frac{1}{2} \int \mathbf{M} \cdot \mathbf{H}^{\prime} \mathrm{d} v
$$

où $\mathbf{M}$ et $\mathbf{H}^{\prime}$ désignent l'intensité d'aimantation et le champ d'excitation en chaque point. L'intégrale est étendue ici à toute la matière aimantée.

Pour se rendre compte de l'importance de ce terme, il suffit d'imaginer une sphère aimantée uniformément à saturation. Prenons l'exemple d'une ferrite avec :

$$
M_{\mathrm{s}}=0,3 \mathrm{~T} \text { et } \mathbf{H}^{\prime}=-\mathbf{M}_{\mathrm{s}} / 3 \mu_{0}
$$

soit

Il vient :

$$
H^{\prime}=8 \times 10^{4} \mathrm{~A} \cdot \mathrm{m}^{-1}
$$

$$
\frac{\mathrm{d} U_{\mathrm{m}}}{\mathrm{d} v}=1,2 \times 10^{4} \mathrm{~J} \cdot \mathrm{m}^{-3}\left(1,2 \times 10^{5} \text { ergs. } \mathrm{cm}^{-3}\right) .
$$

On peut aussi donner de l'énergie magnétostatique une autre expression intéressante.

$$
\mathbf{H}^{\prime} \text { et } \mathbf{B}^{\prime}=\mu_{0} \mathbf{H}^{\prime}+\mathbf{M}
$$

étant deux vecteurs continus et différentiables, l'un irrotationnel, l'autre solénoïdal, nuls à l'infini, on démontre en mathématiques que l'intégrale étendue à tout l'espace :

$$
\int \mathbf{H}^{\prime} \cdot \mathbf{B}^{\prime} \mathrm{d} v=0
$$

Il en résulte :

$$
U_{\mathrm{m}}=\frac{1}{2} \int \mu_{0} \mathbf{H}^{\prime 2} \mathrm{~d} v
$$

L'énergie magnétostatique apparaissant comme une somme de termes tous positifs, on conçoit qu'il y ait intérêt pour diminuer $U_{\mathrm{m}}$ à diminuer les sources du champ $\mathbf{H}^{\prime}$, c'est-à-dire les " masses magnétiques ", et pour des sources données, à limiter l'étendue du champ $\mathbf{H}^{\prime}$.

Qualitativement on diminuera très sensiblement l'énergie magnétostatique de la sphère de ferrite envisagée précédemment si on l'imagine comme un empilement de feuillets aimantés alıernativement dans un sens et dans le sens opposé, bien que dans les deux cas on trouve la même quantité de " masses magnétiques ». Toutefois avec la seconde structure, les lignes de champ restent localisées à la surface de la sphère alors que dans la première structure le volume embrassé par les lignes de champ s'étend beaucoup plus loin (Fig. 1).

En résumé, l'abaissement de l'énergie magnétosta-
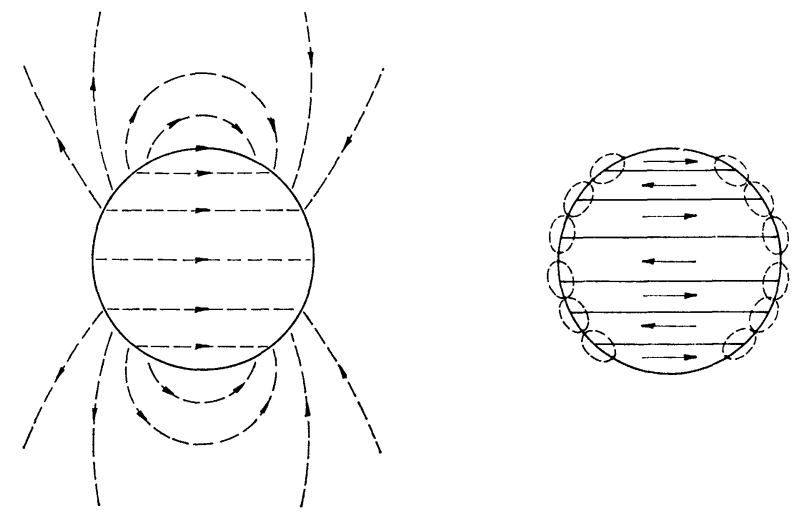

FIG. 1. - Modèle de sphère aimantée uniformément et en feuillets pour diminuer son énergie magnétostatique.

tique peut être obtenu soit en éliminant les sources du champ $\mathbf{H}^{\prime}$, c'est-à-dire les « masses magnétiques » soit en regroupant alternativement des « masses magnétiques ) de signes opposés.

2.3 L'ÉNERGIE D'ANISOTROPIE MAGNÉTOCRISTALLINE. - $\mathrm{Si}$ on imagine un cristal aimanté à saturation c'est-à-dire dont tous les moments atomiques sont parfaitement parallèles, son énergie dépend de l'orientation de $\mathbf{M}_{\mathrm{s}}$ par rapport aux axes du cristal.

C'est un fait d'expérience : Pour la plupart des cristaux magnétiques, il existe des directions de facile et des directions de difficile aimantation.

Par exemple, pour un cristal de ferrite de manganèse-zinc de composition : $\mathrm{MnZn}_{0,2} \mathrm{Fe}_{1,8} \mathrm{O}_{4}$, les axes ternaires sont, à température ambiante, les directions préférentielles pour l'aimantation, et pour amener l'aimantation à saturation dans une direction $<100>$ il faut fournir au cristal environ $300 \mathrm{~J} . \mathrm{m}^{-3}$, ce qui correspond à une anisotropie très faible. C'est une des caractéristiques intéressantes de ce matériau. On connaît d'autres ferrites, par exemple $\mathrm{BaFe}_{12} \mathrm{O}_{19}$ hexagonale, où une rotation de $\pi / 2$ de l'aimantation à température ambiante entre l'axe $c$ et le plan de base demande une énergie 100 fois plus importante.

L'énergie d'anisotropie magnétocristalline est habituellement exprimée en fonction des cosinus directeurs $\left(\alpha_{\mathbf{i}}\right)$ de l'aimantation par rapport aux axes du cristal. Les expressions de la densité d'énergie dépendent de la symétrie du cristal, les cas les plus simples rencontrés étant pour une symétrie uniaxiale :

$$
\mathrm{d} U_{\mathrm{k}} / \mathrm{d} v=-K_{\mathrm{u}} \alpha_{3}^{2}
$$

et pour une symétrie cubique :

$$
\mathrm{d} U_{\mathrm{k}} / \mathrm{d} v=K_{1}\left(\alpha_{1}^{2} \alpha_{2}^{2}+\alpha_{2}^{2} \alpha_{3}^{2}+\alpha_{3}^{2} \alpha_{1}^{2}\right)^{\text {"ै }}
$$

On peut aussi utiliser d'autres développements plus compliqués si ces expressions simples se révèlent insuffisantes à l'expérience. Les coefficients $K_{\mathfrak{u}}, K_{1}$, appelés constantes d'anisotropie du matériau, sont accessibles à la mesure par plusieurs méthodes, soit statique (balance de torsion) (Fig. 2), soit dynamique (résonance ferromagnétique). Enfin, les variations thermiques de l'anisotropie se révèlent souvent 


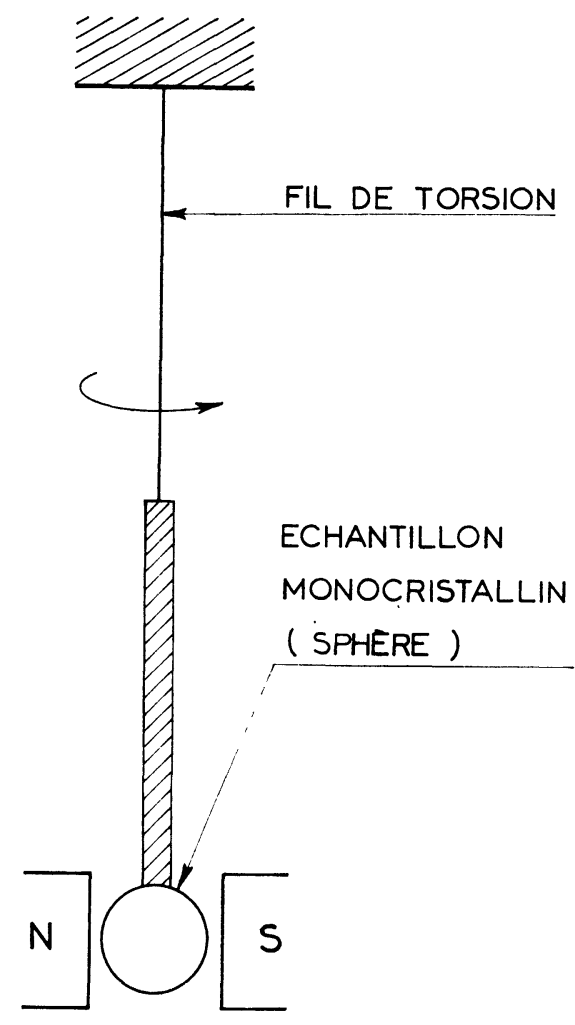

FIG. 2. - Schéma de principe d'une balance de torsion pour la mesure de l'anisotropie magnétocristalline.

importantes et imprévisibles et leur allure étant très variable avec la nature du cristal.

Malgré de nombreux travaux consacrés à la question, on ne dispose pas encore d'une théorie définitive pour rendre compte de l'anisotropie, mais seulement d'une ligne directrice, proposée dès 1931 par Bloch et, pour les métaux ferromagnétiques, par Gentile. Bien que l'interaction d'échange soit isotrope, le moment total verrait le réseau cristallin à cause du couplage entre les moments de spins et les moments orbitaux, ces derniers étant bloqués par le champ cristallin. On comprend dès lors pourquoi les coefficients d'anisotropie les plus importants restent l'apanage des cristaux de basse symétrie. Un calcul de l'anisotropie magnétocristalline nécessiterait dans les composés ioniques une connaissance détaillée du champ cristallin et dans les métaux une connaissance détaillée de la structure de bandes. Les travaux en cours laissent espérer des progrès sensibles dans un avenir proche.

2.4 L'ÉNERGIE MAGNÉTOÉLASTIQUE. — L'énergie magnétoélastique résulte de l'interaction entre la direction de l'aimantation et les déformations du réseau cristallin. Si, quelle que soit la direction de l'aimantation, le réseau cristallin restait rigide, soit parfaitement cubique ou hexagonal il n'y aurait pas d'énergie magnétoélastique.

En pratique l'énergie d'anisotropie magnétocristalline dépend des déformations du réseau cristallin, de sorte que le cristal se déforme spontanément pour réaliser l'état d'énergie libre minimale. Si on imagine par exemple qu'on dispose d'un monocristal parfaitement sphérique dans l'état paramagnétique au-dessus du point de Curie, et qu'après avoir abaissé sa température, on l'aimante à saturation alors le cristal prend spontanément une forme en ellipsoïde.

Limitons-nous au cas simple d'un cristal cubique et appelons $e_{\mathrm{ij}}$ les composantes du tenseur des déformations au nombre de 6. L'énergie interne peut être développée en puissances croissantes des $e_{\mathrm{ij}}$. Compte tenu des symétries du réseau, il vient pour l'énergie libre :

$$
\begin{aligned}
F=U_{\mathrm{k}}+\sum_{\mathrm{i} \geqslant \mathrm{j}} & \left(\frac{\partial F}{\partial e_{\mathrm{ij}}}\right)_{0} e_{\mathrm{ij}}+ \\
& + \text { des termes quadratiques en } e_{\mathrm{ij}}+\cdots .
\end{aligned}
$$

Le premier terme du développement représente l'énergie d'anisotropie magnétocristalline envisagée précédemment. Le second terme constitue l'énergie magnétoélastique et le troisième l'énergie élastique habituelle.

Pour un cristal cubique on trouve que la densité d'énergie magnéto-élastique est donnée par une expression de la forme:

$$
U_{\mathrm{me}}=B_{1} \sum_{\mathbf{j}} e_{\mathrm{ii}}\left(\alpha_{\mathrm{i}}^{2}-\frac{1}{3}\right)+B_{2} \sum_{\mathbf{i} \neq \mathbf{j}} \sum_{\mathrm{ij}} \alpha_{\mathbf{i}} \alpha_{\mathrm{j}}
$$

$B_{1}$ et $B_{2}$ sont les deux constantes de couplage magnétoélastique.

La configuration d'équilibre minimise l'énergie $U_{\text {me }}$ par rapport à chacune des déformations $e_{\mathrm{ij}}$. Par rapport à l'état non déformé, l'allongement relatif du cristal, mesuré dans une direction $\beta_{1} \beta_{2} \beta_{3}$ est alors :

$$
\begin{aligned}
\frac{\Delta l}{l}= & \frac{3}{2} \lambda_{100}\left(\alpha_{1}^{2} \beta_{1}^{2}+\alpha_{2}^{2} \beta_{2}^{2}+\alpha_{3}^{2} \beta_{3}^{2}-\frac{1}{3}\right)+ \\
& +3 \lambda_{100}\left(\alpha_{1} \alpha_{2} \beta_{1} \beta_{2}+\alpha_{2} \alpha_{3} \beta_{2} \beta_{3}+\alpha_{1} \alpha_{3} \beta_{1} \beta_{3}\right) .
\end{aligned}
$$

Les 2 constantes de magnétostriction $\lambda_{100}$ et $\lambda_{111}$ sont respectivement proportionnelles à $B_{1}$ et $B_{2}$ et dépendent aussi des propriétés élastiques du cristal. Enfin si on applique une contrainte extérieure $\sigma$ dans la direction $\left(\gamma_{1} \gamma_{2} \gamma_{3}\right)$ il en résulte une variation de la densité d'énergie

$$
\begin{aligned}
\frac{\mathrm{d}\left(\Delta U_{\mathrm{me}}\right)}{\mathrm{d} v}=-\frac{3}{2} & \lambda_{100} \sigma\left(\alpha_{1}^{2} \gamma_{1}^{2}+\cdots\right)- \\
& -3 \lambda_{111} \sigma\left(\alpha_{1} \alpha_{2} \gamma_{1} \gamma_{2}+\cdots\right) .
\end{aligned}
$$

Ainsi pour $\lambda_{100}>0$, une traction appliquée dans une direction $\langle 100\rangle$ tend à orienter l'aimantation parallèlement à cette traction.

Les coefficients $\lambda_{100}$ et $\lambda_{111}$ sont souvent de l'ordre de $10^{-5}$ et d'accès difficile. Ils peuvent atteindre exceptionnellement $10^{-4}$. En une région du cristal où les contraintes locales valent un kilogramme par millimètre carré $\left(10^{7} \mathrm{~N} . \mathrm{m}^{-2}\right)$ il en résulte une densité 
d'énergie magnétoélastique de l'ordre de $10^{2}$ à $10^{3} \mathrm{~J} . \mathrm{m}^{-3}$, c'est-à-dire comparable à l'énergie d'anisotropie magnétocristalline.

2.5 LeS AUtres ÉNERGIES. - Avec les quatre énergies qui ont été décrites précédemment on dispose de la panoplie complète pour ce qu'on pourrait appeler le cristal parfait. Mais comme chacun sait, la perfection ne se rencontre jamais.

Quand un cristal contient plusieurs sortes d'atomes, il $\mathrm{y}$ a souvent plusieurs configurations possibles d'énergies très voisines pour les mêmes atomes et la distribution d'équilibre dépend de l'orientation de l'aimantation. Il se crée alors par diffusion ce qu'on appelle une surstructure d'orientation. Cet effet est bien connu par exemple dans les ferrites mixtes contenant du cobalt de type $\mathrm{CoFe}_{3} \mathrm{O}_{4}$. Quand un cristal uniformément aimanté est soumis à un traitement thermique, les atomes se redistribuent par diffusion en fonction de son état d'aimantation et par la suite, après retour à température ambiante, on détecte la présence d'une énergie d'anisotropie uniaxiale dans la direction du traitement, énergie dont l'amplitude peut être du même ordre que l'énergie d'anisotropie magnétocristalline dans certaines ferrites. En outre, ces questions de surstructures sont compliquées par des aspects liés à la dynamique des processus de diffusion dans le réseau qui dépend elle-même de la concentration en lacunes.

D'autres défauts de réseau, les disloactions, les joints de grains entraînent certainement beaucoup d'effets difficiles à analyser car ils concernent un moins grand nombre d'atomes. On sait par exemple que des poudres fines de cobalt oxydées puis refroidies sous champ magnétique présentent des cycles d'hystérésis complètement dissymétriques ce qu'on interprète par l'existence d'un couplage unidirectionnel entre la couche d'oxyde superficiel $\mathrm{CoO}$ antiferromagnétique et l'intérieur du grain ferromagnétique.

3. L'existence des domaines. - Nous pouvons maintenant comprendre pourquoi toute substance ferromagnétique isolée se divise spontanément en régions d'aimantation uniforme à une échelle intermédiaire entre l'échelle atomique et le cristal qu'on appelle les domaines élémentaires. C'est simplement un moyen pour abaisser l'énergie interne de l'échantillon.

On peut analyser l'influence des divers mécanismes décrits précédemment en imaginant un cristal ferromagnétique en forme de sphère. En premier lieu, l'énergie d'échange d'une part, et l'énergie magnétostatique d'autre part tendent à faire prévaloir des structures radicalement opposées. L'énergie d'échange, qui est toujours importante, impose en permanence le parallélisme des moments atomiques ce qui signifie qu'à l'échelle atomique le cristal est aimanté à saturation dans les seules directions de facile aimantation afin de minimiser l'énergie d'anisotropie. Mais cette situation correspond à une énergie magnétostatique très importante comme on l'a déjà vu, et c'est donc une configuration habituellement rejetée.

On peut essayer de trouver une configuration plus favorable du point de vue magnétostatique. $\mathrm{Si}$ on imagine une configuration cylindrique de révolution (Fig. $3 A$ ) alors il n'y a pas de "masses magnétiques » libres et par conséquent l'énergie magnétostatique est bien nulle. Mais cette structure s'accompagne à la fois d'une augmentation de l'énergie d'échange qui n'est pas insurmontable car les angles $\varphi_{\mathrm{ij}}$ entre les spins sont faibles et $U_{\mathrm{e}}$ varie comme $\varphi_{\mathrm{ij}}^{2}$ (sauf sur l'axe, mais ça ne concerne que peu d'atomes) et aussi d'un accroissement de l'énergie d'anisotropie a priori peu gênant. Il faut donc chercher autre chose.

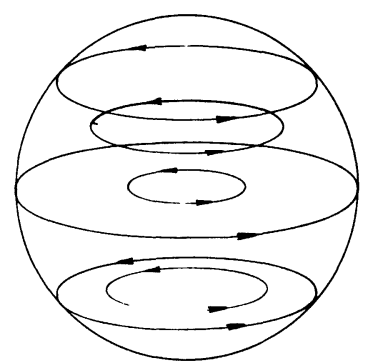

(A)
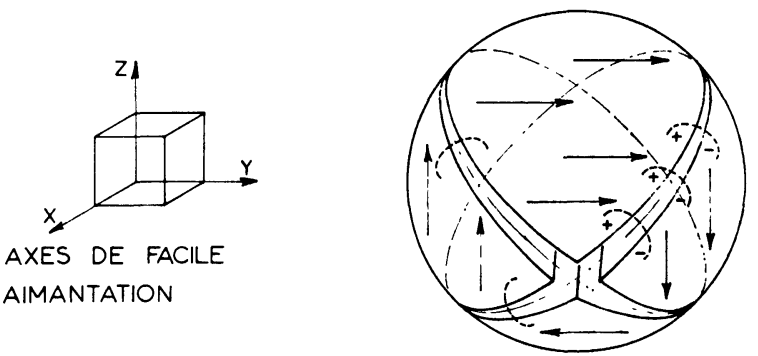

$\lambda>0 \quad$ (B)

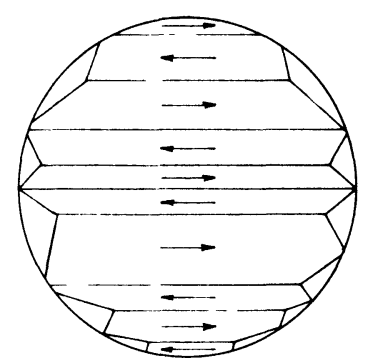

(C)

FIG. 3. - Différentes configurations pour une sphère d'aimantation globale nulle. $A$ ) Symétrie de révolution. $B$ ) Subdivision en quatre domaines aimantés à $90^{\circ}$. C) Subdivision en feuillets antiparallèles.

Imaginons une subdivision en 4 domaines aimantés à $90^{\circ}$ (Fig. $3 B$ ). Il n'y a pas de problème pour l'énergie d'échange sauf aux frontières entre les domaines; c'est parfait aussi vis-à-vis de l'anisotropie magnéto- 
cristalline dans l'hypothèse où les directions d'aimantation des domaines coïncident avec les axes faciles. Mais on voit apparaître deux difficultés du côté de l'énergie magnétostatique et surtout magnétoélastique. En effet si $\lambda>0$ le cristal est allongé dans la direction de $M_{\mathrm{s}}$ et il faut exercer des contraintes pour assurer la continuité de la matière aux frontières entre les domaines.

Un empilement en feuillets alternativement aimantés dans un sens et dans le sens apposé paraît bien préférable et c'est certainement la raison pour laquelle on le rencontre aussi fréquemment. Là encore, la nature s'arrange pour diminuer l'énergie magnétostatique d'une part en augmentant le nombre de feuillets, d'autre part en prévoyant près de la surface des petits domaines additionnels appelés domaines de fermeture (sous-entendu du flux de $\mathbf{M}$ ) (Fig. $3 C$ ).

Ainsi la structure en domaines résulte de nombreuses concessions aux différents mécanismes d'énergie. Pour comprendre les limites de cette subdivision, il faut étudier maintenant la transition entre deux domaines.

4. Les parois. - Il ne peut être question à l'échelle atomique de passer brutalement d'une direction d'aimantation à une autre car une telle transition brutale demanderait une contribution trop importante aux forces d'échange. La zone frontière entre deux domaines s'étend donc sur un certain nombre de plans atomiques qui constituent ce qu'on appelle une paroi (Fig. 4A).

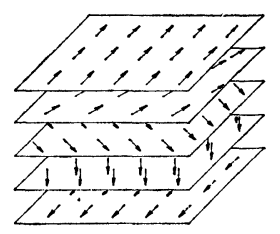

(A)

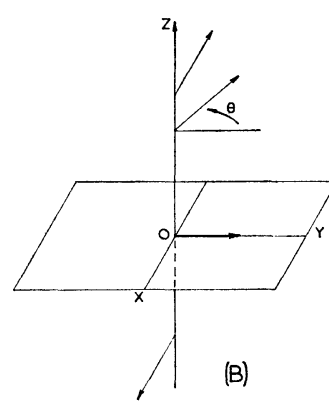

FIG. 4. - Rotation des moments atomiques dans une paroi de Bloch à $180^{\circ}: A$ ) Schéma de paroi. $B$ ) Axes de référence.

4.1 Parol De Bloch a $180^{\circ}$. - Il s'agit d'un problème étudié par $F$. Bloch en 1932. Le problème est celui de la détermination de l'orientation des moments dans la configuration d'équilibre pour un passage graduel par l'intermédiaire d'une série de plans atomiques parallèles.

On suppose l'axe $O Z$ normal au plan de la paroi (Fig. $4 B$ ) et on étudie la rotation des moments dans le plan $(O X, O Y)$ en appelant $\theta$ l'orientation d'un moment à la cote $z$. Les conditions aux limites correspondent à $\theta= \pm \pi / 2$ pour $z= \pm \infty$ et, par raison de symétrie $\theta=0$ pour $z=0$. $\theta$ étant assimilé à une fonction continue de $z$, l'angle $\varphi_{\mathrm{ij}}$ entre 2 spins voisins vaut :

$$
\varphi_{\mathrm{ij}}=\left(\frac{\partial \theta}{\partial z}\right) a
$$

où $a$ est la distance entre les plans atomiques.

L'énergie d'échange entre 2 spins $\mathbf{S}$ voisins est donc de la forme :

$$
W_{\mathrm{ij}}=\frac{J S^{2}}{2} a^{2}\left(\frac{\partial \theta}{\partial z}\right)^{2}
$$

et l'énergie d'échange totale par unité de surface de paroi :

$$
\gamma_{\text {éch }}=\int_{-\infty}^{+\infty} A\left(\frac{\partial \theta}{\partial z}\right)^{2} \mathrm{~d} z
$$

où $A$ est une constante pour un matériau donné. Par ailleurs, l'énergie d'anisotropie par unité de surface vaut :

$$
\gamma_{\mathrm{K}}=\int_{-\infty}^{+\infty} g(\theta) \mathrm{d} z .
$$

La fonction $\theta(z)$ est alors celle qui minimise l'intégrale

$$
\gamma_{\mathrm{t}}=\int_{-\infty}^{+\infty}\left[g(\theta)+A\left(\frac{\partial \theta}{\partial z}\right)^{2}\right] \mathrm{d} z .
$$

C'est un problème classique de calcul des variations et l'équation d'Euler entraîne :

$$
g(\theta)=A\left(\frac{\partial \theta}{\partial z}\right)^{2}
$$

ce qui signifie que pour chaque tranche de la paroi, la densité d'énergie d'anisotropie est égale à la densité d'énergie d'échange.

Ce résultat signifie que, pour une énergie d'échange définie, si l'anisotropie tend vers zéro, alors la rotation des moments atomiques s'effectue très lentement et la paroi de Bloch tend à envahir tout le cristal.

Inversement, dans un matériau à très grande anisotropie les moments tournent rapidement et les parois sont très étroites.

Par intégration de l'équation différentielle (4.2) on obtient la configuration d'équilibre $\theta(z)$ d'où l'on tire l'épaisseur de paroi égale à $\pi \sqrt{A / K_{\mathrm{u}}}$ dans un matériau uniaxe, et l'énergie de paroi correspondante :

$$
\gamma_{\mathrm{t}}=4 \sqrt{A K_{\mathrm{u}}} \text {. }
$$

Ainsi dans le ferrite de baryum $\mathrm{BaFe}_{12} \mathrm{O}_{18}$ Kooy et Enz ont obtenu une confirmation expérimentale remarquable de ce comportement simple [2]. L'énergie de paroi est alors grande : $\gamma_{\mathrm{t}}=28$ ergs. $\mathrm{cm}^{-2}$. Pour une ferrite douce on trouve plus souvent des énergies de paroi de l'ordre de l'erg. $\mathrm{cm}^{-2}$ et des épaisseurs de paroi de l'ordre de la centaine de distances interatomiques.

4.2 ORIENTATION ET RAIDEUR DES PAROIS DE BLOCH. - Le calcul précédent ne prend en compte que les 
énergies d'échange et d'anisotropie. Aucune énergie magnétostatique n'apparaît en effet à l'intérieur de la substance tant qu'il y a conservation de la composante de $\mathbf{M}_{\mathrm{s}}$ normale à la paroi, ce qui privilégie certaines orientations des parois. Mais sur la surface et aux limites du cristal, il en va tout autrement.

En fait des parois parfaitement planes et bien orientées ne se rencontrent pas toujours. A cause des effets magnétostatiques, il est plus facile de " plier » la paroi dans certaines directions un peu comme une tôle ondulée.

4.3 LES AUTRES TYPES DE PAROIS. - On connaît aujourd'hui une grande variété de parois qui se manifestent selon les circonstances, soit parce que les conditions d'observation les favorisent, soit parce que les caractéristiques du matériau imposent une structure particulière. Ainsi par exemple, dans une couche magnétique très mince (une centaine d'angströms dans le fer-nickel) la rotation des moments s'effectue de préférence dans le plan de la couche pour ne pas entraîner la présence d'énergie magnétostatique importante compte tenu du rapport surface sur volume. On a ce qu'on appelle une paroi de Néel [3]. Dans les couches un peu plus épaisses on connaît les parois dites «Cross tie " [4], dans les substances à très forte anisotropie comme $\mathrm{D}_{\mathrm{y}_{3}} \mathrm{Al}_{2}$ les parois dites « étroites » où la rotation des spins s'effectue en quelques plans atomiques seulement [5]. On connaît enfin dans certaines ferrites au cobalt des parois très larges, les « parois $k \pi$ », qui sont liées à une énergie de surstructure importante et dont la structure est en cours d'étude [6].

5. Les méthodes d'observation des domaines. Alors que l'hypothèse d'une subdivision en domaines a été avancée dès 1907 par P. Weiss, il a fallu attendre 1931 pour enregistrer les premières observations par F. Bitter et c'est seulement à la suite des travaux d'Elmore (1938), suivis de ceux de Williams et Bozorth, que la communauté scientifique a été persuadée de la réalité des domaines magnétiques.

Quelle que soit la technique d'observation utilisée, l'analyse d'une configuration en domaines ne peut aboutir que dans les cas les plus simples, avec des cristaux dont l'état de surface est exceptionnellement propre et qui sont exempts de toutes contraintes. L'obtention de tels échantillons est toujours une tâche longue et difficile.

Les méthodes d'observation peuvent être classées en plusieurs groupes.

5.1 LES MÉTHODES INDIRECTES. - Elles reposent sur l'observation d'un paramètre qui dépend de façon indirecte de l'orientation de l'aimantation. Par exemple, au voisinage immédiat de la surface et à l'extérieur d'un cristal, il peut exister un champ magnétique en raison des imperfections de la surface, des impuretés et des parois de Bloch. De nombreuses techniques ont été utilisées pour recueillir des informa- tions sur les domaines à partir de ces champs de dispersion superficiels.

La technique des poudres de Bitter est à la fois la plus ancienne et la plus connue [7]. Elle consiste à déposer sur la surface de l'échantillon une poudre ferromagnétique très fine en suspension colloïdale qui va se rassembler là où le champ est le plus intense. Les traces des parois de Bloch apparaissent alors comme des lignes fines (Fig. 5 et 6).

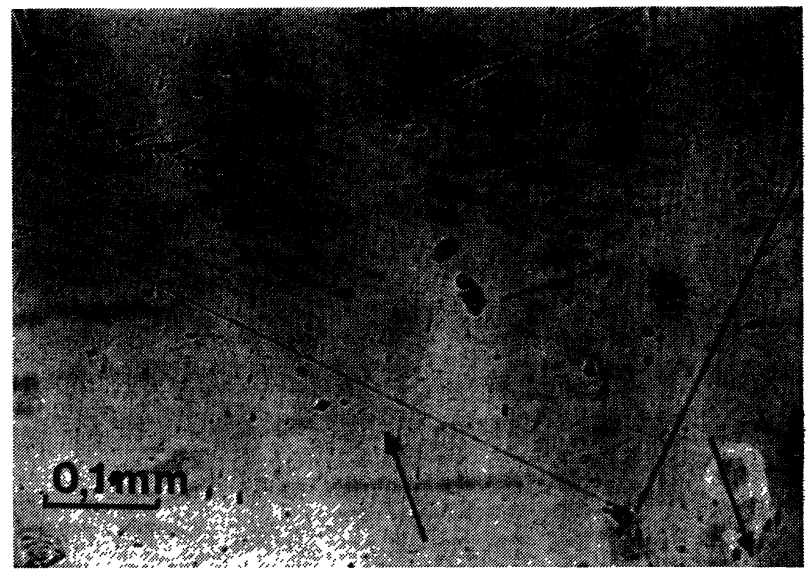

Fig. 5. - Observation de domaines dans un polycristal de fer pur par la technique de Bitter. La ligne courbe en haut du cliché est un joint de grain. Sur le cristal du bas, observé dans un plan (100), on remarque les traces rectilignes des parois de Bloch. La configuration des domaines est ici exceptionnellement simple (R. Aléonard et P. Brissonneau).

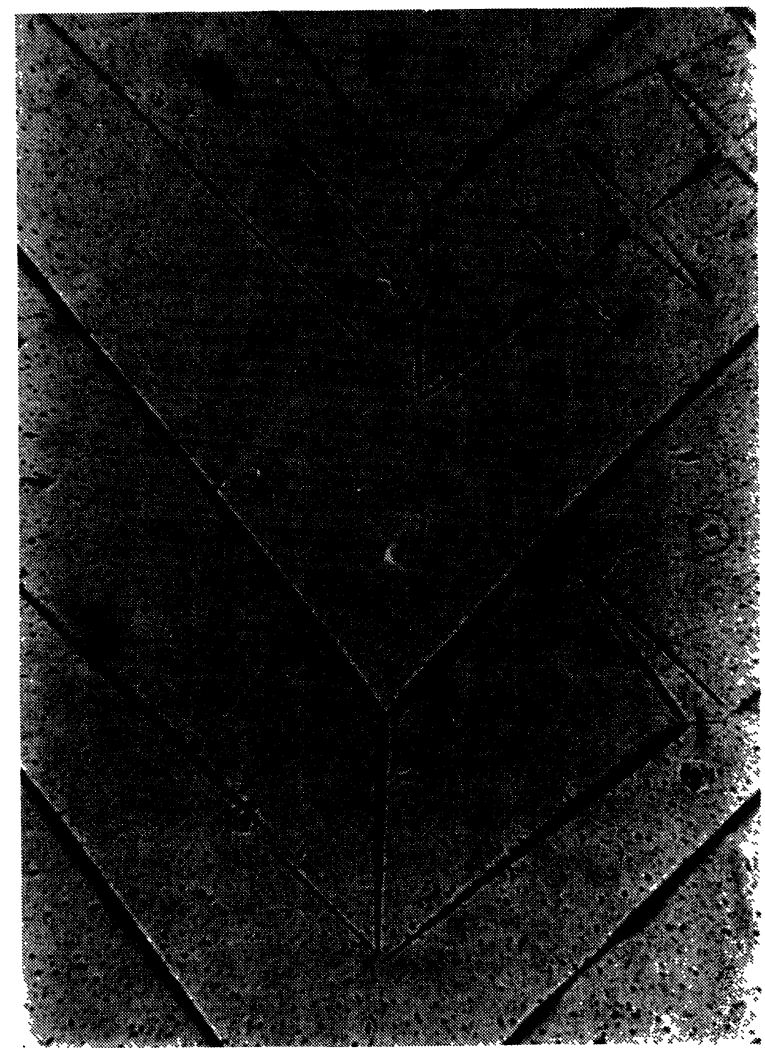

Fig. 6. - Parois de Bloch et domaines de fermeture «en sapins » sur un cristal de fer-silicium. La surface est voisine d'un plan (100). 
De nombreuses variantes ont été signalées où l'étude des champs de dispersion était conduite à l'aide de sondes diverses (permalloy, effet Hall, électrons). D'une façon générale, il faut dire que pour l'observation des domaines et sauf cas d'espèces, les méthodes indirectes apparaissent relativement périmées.

Il faut aussi ranger dans les méthodes indirectes les observations par les méthodes topographiques aux rayons $\mathrm{X}$. Ces méthodes reposent sur la possibilité de mettre en évidence les très faibles rotations du cristal entre deux domaines adjacents consécutives à l'existence de la magnétostriction. Bien entendu cette méthode ne permet pas de déceler les parois de Bloch à $180^{\circ}$ (Fig. 7) que n'accompagne aucune déformation du cristal.

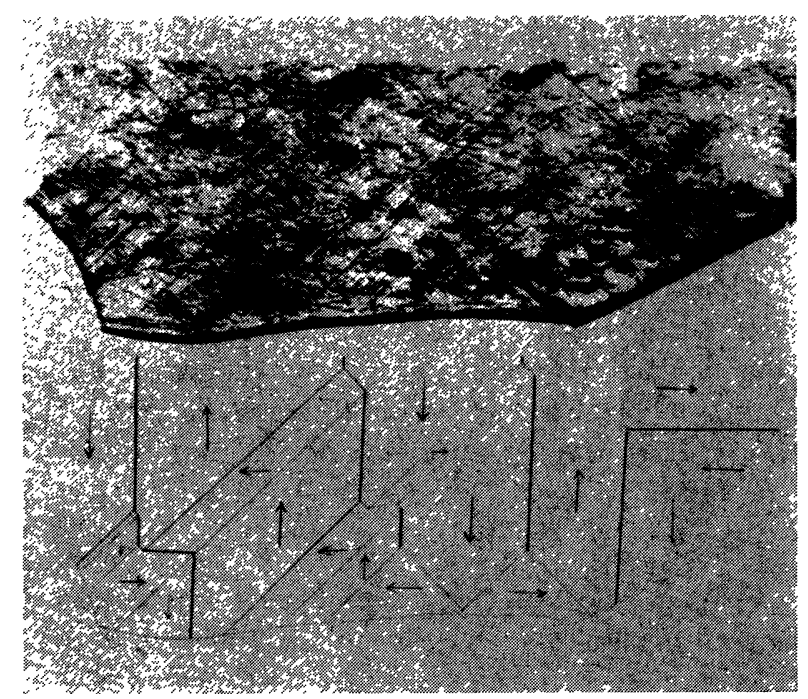

Fig. 7. - Etude de domaines par topographie aux rayons $\mathrm{X}$ dans un cristal de fer-silicium (100) par M. Schlenker (Thèse)

5.2 LeS MÉTHODES DIRECTES. - Un faisceau de particules recueille directement l'information sur l'orientation des moments magnétiques. Il peut s'agir d'électrons, ou de photons, ou encore de neutrons.

La microscopie électronique classique ne permet que l'étude d'échantillons minces (épaisseur allant de 100 à $1000 \AA$ avec un microscope de $150 \mathrm{kV}$ ). Dans la traversée de la matière les trajectoires électroniques sont déviées sous l'effet de la force de Lorentz :

$$
\mathbf{F}_{\mathbf{m}}=e \mathbf{v} \times \mathbf{B} .
$$

On peut alors imaginer plusieurs modes d'observation des domaines et des parois [8]. Les figures 8 et 9 montrent les résultats obtenus par défocalisation sur un film de fer pur d'une part et un film de permalloy d'autre part. D'autres méthodes, moins simples à mettre en œuvre, peuvent conduire à un pouvoir séparateur bien meilleur.

Les méthodes optiques ont connu un très grand développement durant ces dernières années. Un fais-

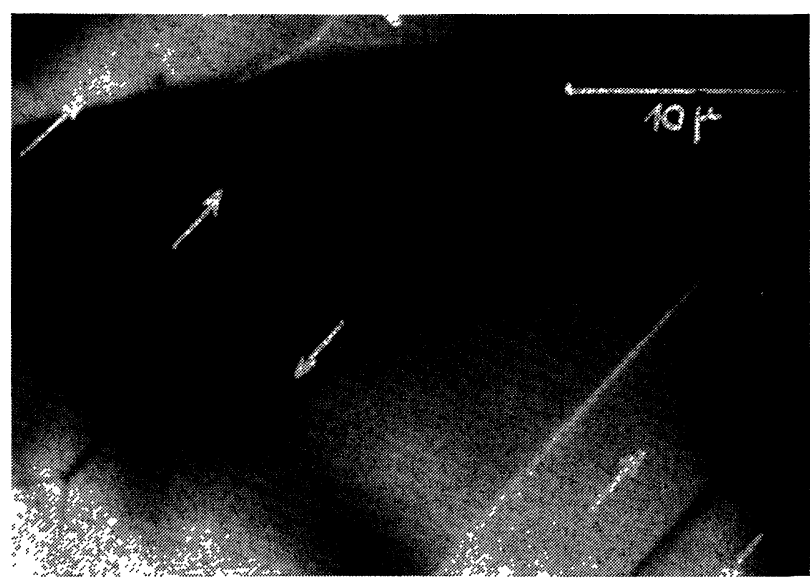

FIG. 8. - Domaines observés dans une lame mince (100) de fer pur en microscopie de Lorentz par A. Bourret.

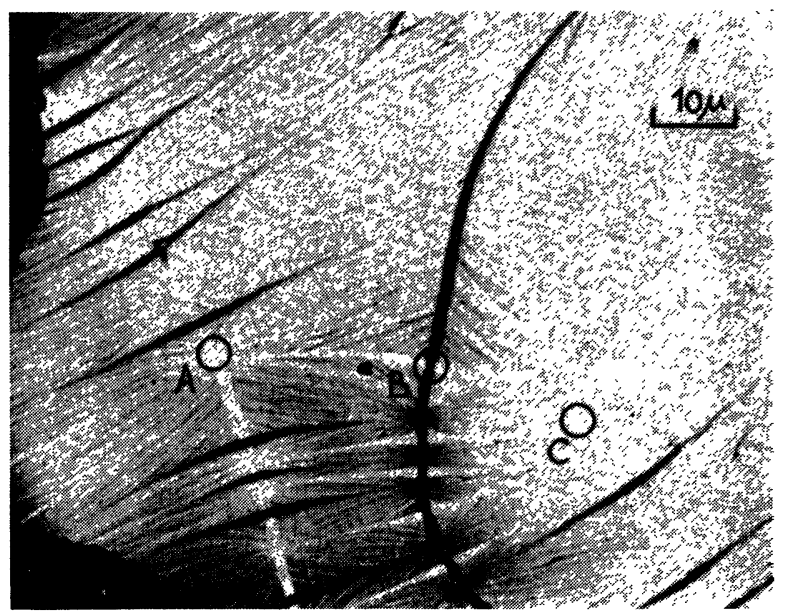

Fig. 9. - Domaines et parois "Cross-tie" dans un film de permalloy. Etude en microscopie de Lorentz par R. H. Wade [8].

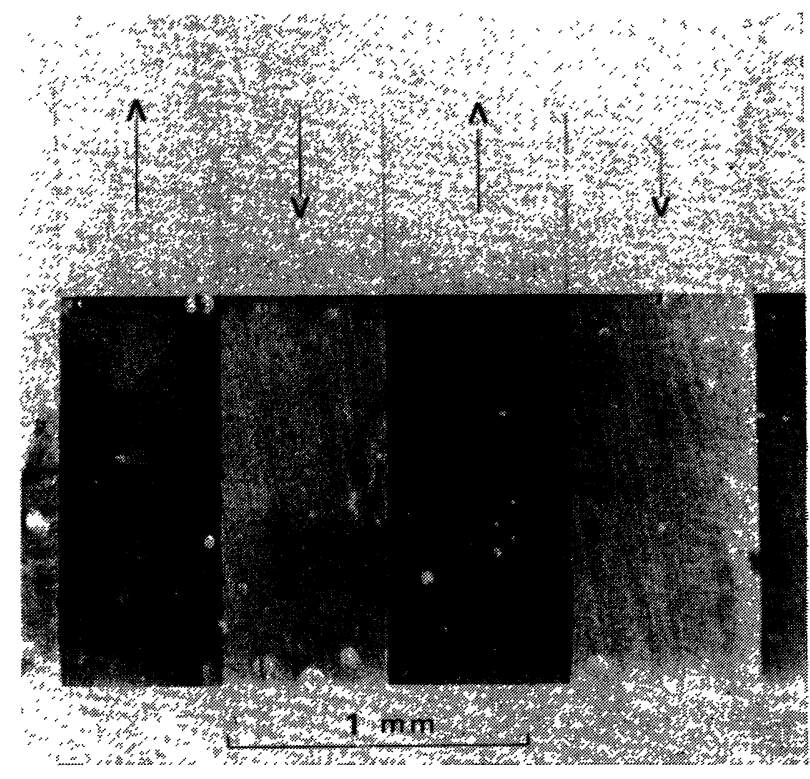

Fig. 10. - Domaines à $180^{\circ}$ observés par effet Kerr longitudinal sur un cristal de fer (100). 
ceau de lumière parallèle polarisée rectiligne interagit avec le milieu magnétique et il en résulte une rotation du plan de polarisation de la lumière. Malheureusement assez peu de matériaux magnétiques sont assez transparents pour qu'on puisse observer les domaines magnétiques en lumière transmise (effet Faraday). C'est toutefois le cas du célèbre grenat d'yttrium et d'un certain nombre d'isolants. Dans le cas des matériaux réflecteurs, on détecte la rotation du plan de polarisation du faisceau réfléchi (effet Kerr). On sait détecter des rotations faibles de quelques minutes d'arc seulement (Fig. 10 et 11). Il faut insister sur le fait que les méthodes optiques d'observation des domaines présentent des avantages très notables, en particulier elles donnent une information sûre et directe sur l'orientation de l'aimantation. Elles sont également faciles à mettre en œuvre à toute température et assez peu coûteuses.

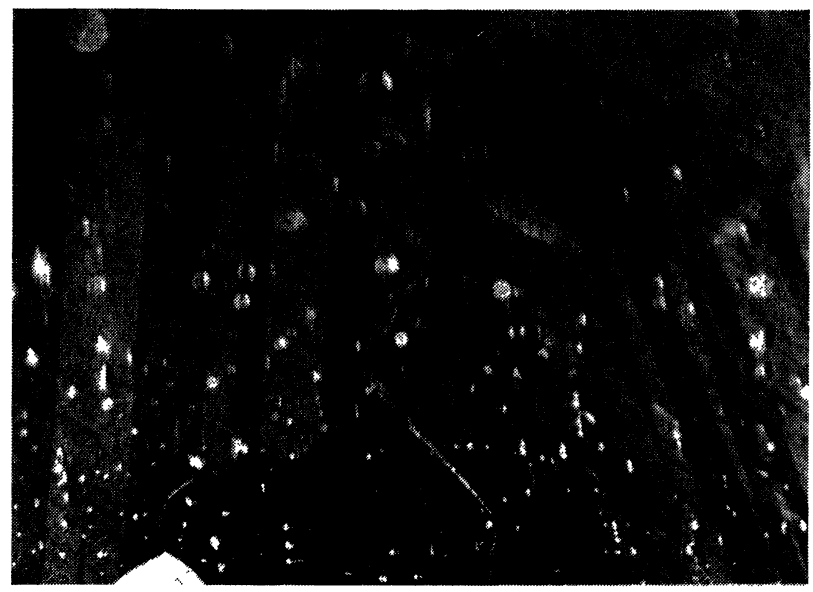

FIG. 11. - Domaines sur un échantillon de FeSi à grains orientés polycristallins (texture Goss). Observation par effet Kerr longitudinal. Noter les directions de facile aimantation et la structure en domaines variables d'un cristal à l'autre.

6. Généralités sur les configurations de domaines. Il nous faut insister maintenant sur le fait que sauf cas particulier on ne sait pas dire a priori comment la matière va exactement se subdiviser en domaines afin d'abaisser son énergie totale. Jusqu'ici nous avons raisonné à partir de modèles intuitifs qui dépendent beaucoup des facultés d'imagination du physicien qui les propose. Les modèles qualitatifs simples ne prennent en considération que deux ou trois types d'énergie, ce qui est notoirement insuffisant pour rendre compte des détails, en particulier au voisinage immédiat des limites du cristal et des défauts du réseau. La détermination d'une configuration générale d'énergie minimale est un problème encore trop complexe pour les ordinateurs de la génération actuelle. En outre, le problème ainsi posé n'aurait guère de signification car les configurations réelles ne réalisent pas des minima d'énergie absolus c'est-à-dire qu'à des conditions extérieures déterminées peuvent correspondre plusieurs configurations en domaines distinctes qui se manifestent par l'existence de l'hystérésis.
7. Les domaines et les processus d'aimantation. 7.1 LeS ROTATIONS. - La structure en domaines étant admise, on peut envisager plusieurs processus d'aimantation distincts. Par exemple, on peut imaginer que les moments atomiques, initialement dans une direction de facile aimantation, s'écartent progressivement de leur position initiale sous l'effet d'un champ extérieur. C'est un processus de rotation cohérente à l'échelle de l'ensemble des moments d'un même domaine. Il apparaît évidemment d'autant plus facilement que l'anisotropie du matériau est plus faible et sa caractéristique principale réside dans le fait que les moments reviennent à leur position initiale dès que le champ moteur disparaît, ce qu'on caractétise en disant qu'il s'agit d'un processus d'aimantation réversible.

7.2 LeS DÉPLACEMENTS DE PAROIS. - Il peut y avoir aussi sous l'effet d'un champ appliqué, une rotation des moments à l'intérieur des parois et dans leur voisinage immédiat, équivalente à un déplacement des parois. Certains domaines voient leur volume augmenter au détriment d'autres domaines moins favorisés. Il s'agit là d'un processus d'aimantation très important dans les matériaux magnétiques faciles à aimanter. Il suffit souvent d'un champ très réduit pour déplacer certaines parois et la suppression de ce champ n'entraîne pas obligatoirement le retour des parois dans leur position initiale, d'où l'origine de l'hystérésis.

7.3 Modèle a une SeUle Paroi. - Pour simplifier notre compréhension des mécanismes d'aimantation, on peut imaginer le cas idéal où une seule paroi sépare deux domaines contigus. L'effet d'un champ interne $\mathbf{H}_{\mathrm{i}}$ appliqué équivaut alors à une pression sur la paroi comme il résulte du théorème des travaux virtuels.

Si $\mathbf{M}_{\mathbf{s}_{1}}$ et $\mathbf{M}_{\mathbf{s}_{2}}$ sont les 2 directions d'aimantation dans les domaines de part et d'autre de la paroi, tout déplacement $\mathrm{d} z$ de la paroi entraîne une variation d'énergie par unité de surface :

$$
\mathrm{d} W=-\mathbf{H}_{\mathbf{i}}\left(\mathbf{M}_{\mathrm{s}_{1}}-\mathbf{M}_{\mathrm{s}_{2}}\right) \mathrm{d} z .
$$

D'où la pression motrice de type hydrostatique :

$$
P_{\mathrm{i}}=\mathrm{H}_{\mathrm{i}} \cdot \mathbf{M}_{\mathrm{si}} \text {. }
$$

L'effet du champ appliqué dépend donc de la configuration en domaines et on conçoit sans peine l'intérêt qu'il peut y avoir en pratique à favoriser certaines orientations privilégiées des domaines, ce qu'on peut réaliser en induisant une surstructure d'orientation par exemple.

Ce comportement est illustré sur la figure $12 B$. Sous l'effet du champ appliqué parallèlement $\mathrm{au}$ grand côté de la plaquette, on observe très nettement la courbure de la paroi à $180^{\circ}$ bloquée à ses deux extrémités par les domaines de fermeture. Cette paroi évoque une membrane de tension superficielle $\gamma$ soumise à une pression $P$. 
A

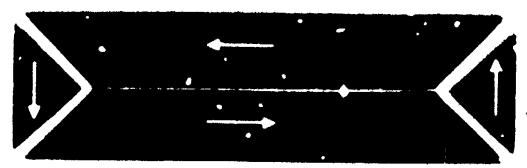

B

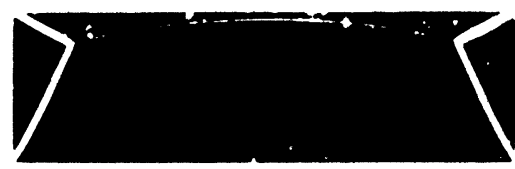

C

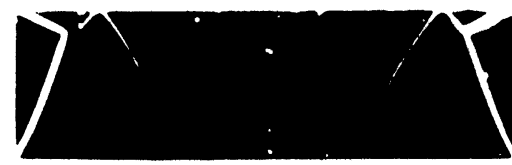

D

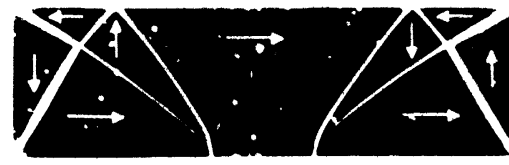

$E$

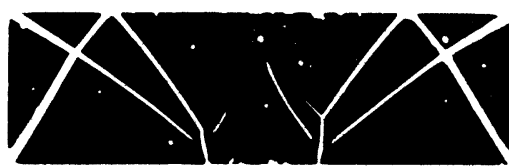

$\mathbf{F}$

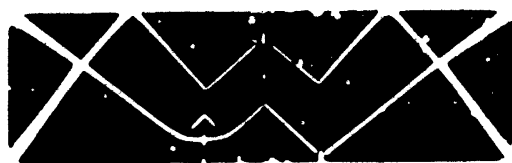

G

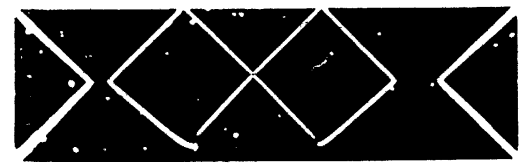

Fig. 12. - Etude de domaines sous champ magnétique appliqué parallèlement au grand côté d'une plaquette rectangulaire de Ni-Co. Il s'agit d'un cristal pratiquement parfait, de type « wiskers » et de dimensions : $\left.112 \times 35 \times 0,55(\mu . \mathrm{m})^{3} . A\right) H=0$, configuration d'équilibre. $B$ ) $H=5,1 \mathrm{Oe}$, noter la courbure de la paroi à $180^{\circ}$ ancrée à ses 2 extrémités par les domaines de fermeture. $C) H=6,0 \mathrm{Oe}$, la paroi à $180^{\circ}$ disparaît. $\left.D\right) H=1,8 \mathrm{Oe}$, la configuration initiale ne réapparaît pas spontanément quand le champ décroît. $E$ ) et $F$ ) $H=1,4 \mathrm{Oe}$, on observe une modification spontanée dans l'arrangement des domaines. $G) H=0$, on retrouve une configuration métastable. D'après $R$. W. De

Blois G. E. Laboratory Rep. no 65-C-082 (Déc. 1965).

7.4 MÉCANISME DU DÉPLACEMENT D'UNE PAROI ISOLÉE. - Les moments magnétiques soumis à un champ extérieur obéissent à une équation du type de celle proposée par Gilbert (1955) :

$$
\frac{\mathrm{d} \mathbf{M}}{\mathrm{d} t}=\gamma_{0} \mathbf{M} \times\left(\mathbf{H}_{\mathrm{eff}}-\eta \frac{\mathrm{d} \mathbf{M}}{\mathrm{d} t}\right)
$$

où $\gamma_{0}$ est le rapport gyromagnétique et $\mathbf{H}_{\text {eff }}$ le champ effectif appliqué. La première partie du second membre rend compte de la précession des moments autour du champ $\mathbf{H}_{\text {eff }}$ et le second terme d'un effet d'amortissement, de sorte que tout se passe comme si le système des moments effectuait un mouvement de précession amorti par un frottement visqueux.

Quand on applique un champ dirigé suivant $O X$ à une paroi simple à $180^{\circ}$ comme celle qui est représentée figure $4 B$, seuls les moments intérieurs à la paroi sont mis en mouvement de précession autour de $O X$. Ce mouvement fait immédiatement apparaître un champ local parallèle à $O Z$, soit perpendiculaire au champ initial et analogue à un champ démagnétisant. C'est la précession des moments autour de ce $2^{\mathbf{e}}$ champ qui rend compte du déplacement de la paroi. Le traitement rigoureux du problème apparaît de ce fait assez compliqué. La dynamique des parois a fait l'objet encore récemment de plusieurs travaux [9-10-11] et on peut résumer l'état de la question de la façon suivante :

7.4.1 Dans les champs faibles, il existe un régime stationnaire pour lequel la vitesse de paroi varie linéairement avec le champ appliqué (Fig. 13) :

$$
v=\alpha\left(H-H_{\mathrm{c}}\right) \text {. }
$$

Dans cette expression $\alpha$ représente la mobilité de la paroi et $H_{\mathrm{c}}$ un champ coercitif propre à la paroi, dû aux impuretés, aux domaines de fermeture et aux conditions aux limites.

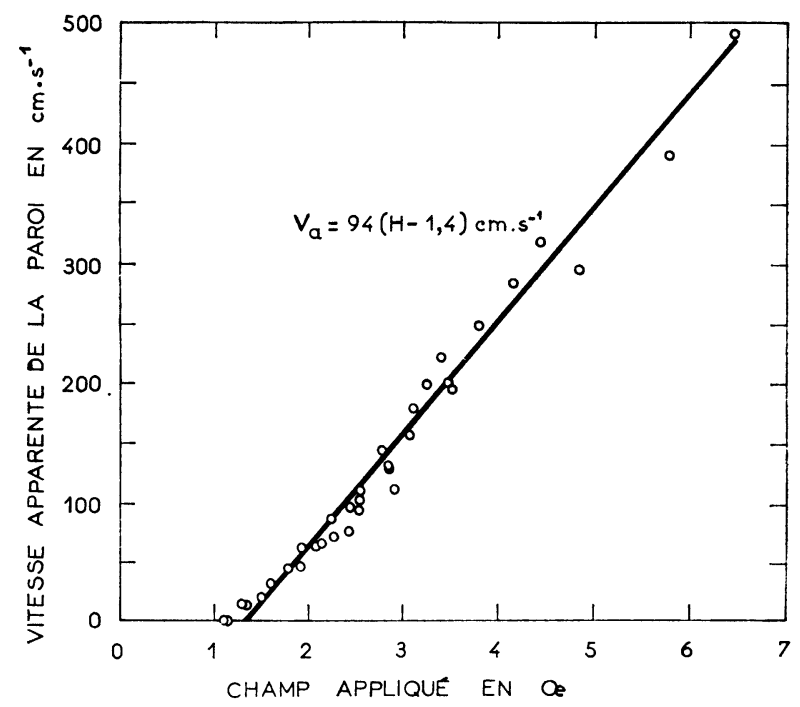

Fig. 13. - Vitesse de paroi en fonction du champ appliqué dans un monocristal $\mathrm{Mn}_{1,4} \mathrm{Fe}_{1,6} \mathrm{O}_{4}$ à $T=20 \mathrm{~K}$. D'après Dillon, J. F. et Earl, H. E., J. Appl. Phys. 30 (1959) 202.

Dans les matériaux conducteurs, la mobilité $\alpha$ reste limitée par l'existence des courants induits (à des valeurs de l'ordre $\mathrm{du} \mathrm{cm} / \mathrm{s} . \mathrm{Oe}$ ). Dans les isolants, la mobilité peut atteindre des valeurs nettement plus élevées mais elle reste encore finie à cause du couplage entre les moments et le réseau (éq. (7.1)).

En régime stationnaire, la paroi en mouvement acquiert en raison de sa structure interne un supplément d'énergie proportionnel au carré de sa vitesse. En d'autres termes tout se passe comme si elle possédait une énergie cinétique $\frac{1}{2} m v^{2}$, avec une masse très faible de l'ordre de $10^{-10} \mathrm{~g} . \mathrm{cm}^{-2}$. 
7.4.2 Un certain nombre de dispositifs à mémoires sont basés sur des configurations de domaines magnétiques, en particulier les bulles. Les utilisateurs sont intéressés par des déplacements de parois aussi rapides que possible. Quand on augmente le champ moteur on trouve qu'il existe une vitesse critique audelà de laquelle le déplacement donne naissance à des ondes de spin. Les vitesses critiques observées sont de l'ordre de quelques dizaines de $\mathrm{m} . \mathrm{s}^{-1}$, de sorte qu'un renversement d'aimantation par déplacement de paroi demande un temps au minimum de l'ordre de la microseconde.

7.5 Aimantation en Champ faible. - Dans le domaine des aimantations très faibles (de l'ordre de $10^{-2} M_{s}$ ), l'aimantation résulte la plupart du temps de petits déplacements des parois sur des distances très inférieures à la largeur des domaines de sorte que la configuration d'ensemble des domaines n'est pas sensiblement modifiée. Quand le champ moteur est supprimé les parois reviennent pratiquement à leur position de départ et c'est pourquoi on parle quelquefois d'aimantation réversible. Dans un modèle à une paroi en équilibre dans un puits de potentiel parabolique, la perméabilité magnétique dépend simplement de la surface de paroi et de la forme du puits de potentiel.

7.6 HYSTÉRÉSIS ET CHAMP COERCITIF. - Dans les champs moyens où les processus d'aimantation acquièrent leur complexité maximale, l'aimantation obtenue dépend de la subdivision en domaines avec plusieurs configurations en domaines possibles pour des conditions extérieures données. Les considérations énergétiques ne peuvent pas tout expliquer comme le montre à l'évidence l'exemple de la figure $12, A$ à $G$. Le domaine d'aimantation inverse détruit par l'application d'un champ de 6 Oe ne se reforme pas spon- tanément même dans un champ nul. En d'autres termes il $\mathrm{y}$ a un problème de nucléation qui intéresse le matériau à une échelle bien inférieure à celle des domaines. On parle alors de « micromagnétisme » [12]. D'une façon générale, la nucléation des domaines d'aimantation inverse est à la base du comportement d'un certain nombre d'aimants en grains fins. Les défauts de réseau, comme les dislocations, les impuretés, les limites du cristal se comportent dans certains cas comme autant de centres de nucléation privilégiés. Mais on connaît aussi des exemples d'un comportement inverse où les défauts freinent jusqu'au blocage les déplacements de paroi. En résumé, le champ coercitif peut apparaître soit comme un champ de nucléation moyen des domaines inverses, soit comme le champ seuil moyen nécessaire pour que les parois balaient les domaines.

8. Conclusion. - Les domaines magnétiques représentent une structure à une échelle intermédiaire entre l'ensemble du cristal et l'échelle atomique. Leur existence est un fait acquis. On sait les mettre en évidence et on a pu étudier leur comportement dans quelques cas simples.

On a fait une grande part au début de cet article aux différentes énergies d'interaction qui permettent d'expliquer la présence des domaines. Ces énergies d'interaction permettent d'imaginer un bon nombre de structures complexes mais l'obtention de structure même statique dépend de problèmes dynamiques compliqués, et encore incomplètement résolus.

D'un point de vue pratique, la connaissance des domaines doit permettre de faire progresser la science des matériaux, de préparer des matériaux aux performances améliorées et d'utiliser avec plus de profit les matériaux existants.

\section{Bibliographie}

[1] Kittel, C., Physical theory of felromagnetic domains, Rev. Mod. Phys. 21 (1949). Cet exposé, considéré aujourd'hui comme classique, a été reproduit dans de nombreux ouvrages en particulier :

KitTeL, C., Introduction à la physique de l'état solide (Dunod Edit.) 1970 ;

Chikazumi, S., Physics of magnetism (J. Wiley) 1964.

[2] Kooy, C. and Enz, U., Philips Research Repts. 15 (1960) 7.

[3] Néel, L., C. R. Hebd. Séan. Acad. Sci. 241 (1955) 533.

[4] Huber, E. E., Smith, D. O. et Goodenough, J. B., J. Appl. Phys. 29 (1958) 294.

[5] BARbara, B., J. Physique 34 (1973) 1039.

[6] Simonet, W. et Paulus, M., J. Physique 28 (1967) 653.
[7] Craik, D. J. et Tebble, R. S., Ferromagnetism and Ferromagnetic domains (North Holland Pub. Cy, Amsterdam) 1965.

[8] Wade, R. H., J. Physique Collq. 29 (1968) C2-95.

[9] FeldtKeller, E., Phys. Stat. Sol. 27 (1968) 161-170.

[10] SchlömanN, E., A. I. P. Conference Proceedings, $n^{0} 5$, p. $160-164$ (1971).

[11] Bourne, H. C. and Bartan, D. S., I. E. E. E. Trans. Magn. Mag $8 n^{\circ} 4,741-746$.

[12] BRown, W. F., Magnetostatic principles in ferromagnetism (North-Holland) 1962. Traduction française publiée dans les Monographies Dunod «Principes de ferromagnétisme ». 